\title{
English-Chinese Translation Strategies for Product Instructions
}

\author{
Lu Feng* \\ Donghua University, Shanghai 200000, China \\ *Corresponding author: Lu Feng, carolinefeng1997@163.com
}

\begin{abstract}
The exchange between domestic and overseas products as well as services is increasing owing to the gradual advancement of globalization. In 1993, Gu Rong first proposed the concept of "translating product instructions," then product instructions became a hot topic in the field of translation research. Based on empirical research, this paper analyzes the textual characteristics and translation strategies for product instructions in a bid to provide reference for translation research ${ }^{[1]}$.
\end{abstract}

Keywords: English-Chinese translation; Translation strategy; Product instructions

Publication date: December 2021; Online publication: January 24, 2022

\section{Introduction}

Due to the differences between Chinese and English, translators face various difficulties in the process of English-Chinese translation. Compared with other types of text, especially literary text, product instructions aim to make factual descriptions without subjective feelings ${ }^{[2]}$. Eugene Nada emphasizes on "content first, the form" in Functional Equivalence Theory, undoubtedly exerting illuminative impact on translators in their translation of product instructions.

\section{Translation strategies}

The types of sentences in product instructions can be mainly divided into passive sentences, elliptical sentences, as well as long and difficult sentences.

A passive sentence involves a passive relationship between the subject and object, which is not only conducive to highlighting the theme of paragraphs and promoting the flow of information, but also emphasizing the receiver to avoid the focus on the agent ${ }^{[3]}$. Chinese people are not familiar with using the passive voice, so translators need to add the subject as the agent or transform the passive relationship into an active relationship to make a smooth convergence.

An elliptical sentence stresses the key information and avoids repetition. Despite the fact that various tenses and plural forms can be omitted in view of Chinese writing habits, translators should look at parallel texts and understand the background information, especially to master synonyms and hypernyms of words, so as to understand the complete meaning of those texts. However, translators can complete the information and make a text understandable by clearly translating strong verbs in English.

A long and difficult sentence involves many modified components. English is hypotactic language with strict sentence structure, and important information is always placed first, whereas Chinese is a paratactic language with a relatively loose sentence structure, and non-important information is usually placed in front [4]. Therefore, in the process of English-Chinese translation, translators need to ensure the content structure is reasonable and logically accepted by Chinese readers in accordance to the differences. 


\section{Conclusion}

Product instructions are texts with "functional style," involving passive sentences, elliptical sentences, as well as long and difficult sentences. They emphasize on the coherence and logic of the text. Therefore, faced with the technical and supporting details of English-Chinese product instructions, translators need to refine their translation through constant accumulation of experience and solve the problems of different product instructions.

\section{Disclosure statement}

The author declares that there is no conflict of interest.

\section{References}

[1] Feng Q, Wang W, 2019, Problems in Translation of Product Specification and Its Countermeasures. Read and Write (Educational Journal), 16(01): 2, 4.

[2] Lai X, 2019, Translation of Mechanical Product Use Instructions. Modern Communication, 2019(06): 98-100.

[3] Wang W, 2019, Tech Product Specification Under the Theory of Functional Peers, English-Chinese Translation Practice Report, West China University of Technology.

[4] Wang JN, 2018, The Use of the Product Instructions Under the Functional Peer-To-Theoretical, Southeast University. 